\title{
Comparative Effects of Monosialoganglioside versus Citicoline on Apoptotic Factor, Neurological Function and Oxidative Stress in Newborns with Hypoxic-Ischemic Encephalopathy
}

\author{
Shi-Peng Liang1', Qian Chen², Yi-Bing Cheng1', Ying-Ying Xue ${ }^{1}$ and Hai-Jun Wang ${ }^{1}$
}

\begin{abstract}
Objective: To determine the comparative effect of monosialoganglioside versus citicoline on the content changes of serum apoptotic factors (PDCD5, sFas and sFasL), neurological function indices (BDNF, NSE, S100- $\beta$ and NGF) and oxidative stress indices (SOD, MDA and GSH-PX) in newborns with hypoxic-ischemic encephalopathy (HIE).

Study Design: An experimental study.

Place and Duration of Study: Emergency Department, Affiliated Children's Hospital of Zhengzhou University, China, from October 2016 to February 2018.

Methodology: A total of 90 newborns with HIE were randomly divided into a treatment group and a control group, with 45 cases in each group. In addition to the conventional treatment, the treatment group was given monosialoganglioside treatment, while the control group was given citicoline treatment. Both groups were treated for 10 days. After treatment, the content differences of serum apoptosis factors (PDCD5, sFas and sFasL), neurological function indices (BDNF, NSE, S100- $\beta$ and NGF) and oxidative stress indices (SOD, MDA and GSH-PX) were observed in the two groups.

Results: After treatment, the levels of serum PDCD5, sFas, sFasL, MDA, NSE and S100- $\beta$ in the treatment group were lower than those in the control group (all $p<0.001$ ). The contents of serum SOD, GSH-PX, BDNF and NGF in the treatment group were higher than those in the control group (all $p<0.001$ ).

Conclusion: Monosialoganglioside can effectively improve the apoptotic factors, neurological function and oxidative stress indices in newborns and maintain the stability of the internal environment, so it is worthy of promotion and application.
\end{abstract}

Key Words: Newborn, Hypoxic-ischemic encephalopathy (HIE), Monosialoganglioside, Citicoline.

\section{INTRODUCTION}

Hypoxic-ischemic encephalopathy (HIE) is mainly caused by intrauterine hypoxia and partial or complete hypoxia caused by perinatal asphyxia, fetal or neonatal brain injury caused by cerebral blood flow reduction or pause..$^{1,2} \mathrm{HIE}$ is not only a serious threat to the life and health of newborns, but also the most common cause of cerebral palsy, mental retardation, hearing impairment, and epilepsy in children. Itoo et al. pointed out that the important associated risk factors of HIE included being a primigravida mother, lack of antenatal care, pregnancy induced hypertension, prolonged 2nd stage of labor, delivery by use of instruments or emergency cesarean section and intrauterine growth retardation. ${ }^{3}$ Early, timely and effective treatment can improve the cure rate of children with HIE, reduce the mortality rate of children

1 Department of Emergency, Affiliated Children's Hospital of Zhengzhou University, Zhengzhou, 450000, China

2 Department of Clinical Medicine, Xinxiang Medical University, Xinxiang, 453003, China

Correspondence: Shi-Peng Liang, Department of Emergency, Affiliated Children's Hospital of Zhengzhou University,

Zhengzhou, 450000, China

E-mail:rpscy3@163.com

Received: June 09, 2018; Accepted: October 30, 2018 and improve the prognosis of the nervous system. However, the condition of HIE is complex and serious, and the pathogenesis is not yet completely clear. Therefore, HIE treatment has not achieved breakthrough progress, and there is no unified effective treatment with the use of drugs and other interventional methods, but mainly based on comprehensive supportive and symptomatic care. 4,5 Medical therapy is an important part of HIE treatment. Therapeutic agents include barbiturates, allopurinol, magnesium sulfate, mannitol, naloxone, dopamine and others isoflourine, allopurinol, erythropoeitin. However, there is no clinical evidence that these drugs can significantly reduce the mortality rate of children with HIE and improve the prognosis of the nervous system.6,7 How to choose drugs needs to be further studied.

Monosialoganglioside is a widely used drug for the treatment of nervous system diseases. It has a good curative effect on vascular or traumatic central nervous system injury and senile dementia. It can also be used as an enhancer of neurotrophic factors to promote the recovery of neurological function. Clinically, it is mainly used in the treatment of acute ischemic stroke, primary brain stem injury, acute spinal cord injury, and peripheral nerve injury. ${ }^{8}$ Animal experiments have also confirmed that monosialoganglioside has neuroprotective effects 
on rats with neonatal hypoxic-ischemic brain damage, which can reduce the occurrence of cerebral edema and convulsions, promote the recovery of brain damage, and increase learning and memory function of aged rats with neonatal hypoxic-ischemic brain damage. 9 Citicoline (CDP-choline; cytidine 5'-diphosphocholine), is a nucleic acid derivative, and it belongs to the precursor substance of phosphatidylcholine and coenzyme biosynthesized by lecithin.10 Citicoline has been evaluated in animal experiments and human clinical trials that provide evidence of its cholinergic and neuroprotective actions. ${ }^{11}$ Some studies have suggested that citicoline can reduce cerebral vascular resistance, increase cerebral blood flow perfusion, reduce cerebrovascular palsy and cerebral edema, and promote the recovery of brain tissue. ${ }^{12}$

The objectives of this study was to determine the comparative effect of monosialoganglioside versus citicoline on the content changes of serum apoptotic factors (PDCD5, sFas and SFasL), neurological function indices (BDNF, NSE, S100- $\beta$ and NGF) and oxidative stress indices (SOD, MDA and GSH-PX) in newborns with hypoxic-ischemic encephalopathy (HIE).

\section{METHODOLOGY}

This study was conducted at the Emergency Department, Affiliated Children's Hospital of Zhengzhou University, China, from October 2016 to February 2018. A total of 90 newborns with HIE, meeting the criteria of Diagnostic Basis and Clinical Grading of Neonatal Hypoxicischemic Encephalopathy formulated by China in 1996, all grades, were included. The diagnosis of brain damage was confirmed by CT brain examination and it met the diagnostic criteria for HIE, established by the World Health Organization (WHO) reference standard; in absence of other severe congenital organ dysfunction. The family members of children understood the research process and signed the informed consent. Exclusion criteria were congenital cerebral vascular dysfunction; interruption of treatment or family members voluntarily waiving treatment; and incomplete clinical data.

The 90 newborns were randomly divided into a treatment group and a control group by using a random number table, with 45 cases in each group. Both groups were given the conventional basic treatment, including reducing intracranial pressure, controlling convulsions, providing the daily fluid requirement, correcting hypotension, maintaining blood glucose in the normal range, and maintaining water and electrolyte balance. In the control group, $125 \mathrm{mg}$ citicoline was added to $20 \mathrm{~mL}$ of $5 \%$ glucose injection and infused once a day for 10 days. The treatment group received monosialoganglioside on the basis of routine treatment, i.e., $20 \mathrm{mg}$ monosialoganglioside was added to $20 \mathrm{~mL}$ of $5 \%$ glucose injection and infused once a day for 10 days. After 10 days of treatment in both the groups, one $\mathrm{ml}$ of blood was collected and left to stand at room temperature and centrifuged to remove the supernatant for serological detection. The content of programmed cell death gene 5 (PDCD5), soluble Fas (sFas), and soluble Fas ligand (sFasL) were determined by ELISA double antibody sandwich method. Neurological function indices, i.e. brain-derived neurotrophic factor (BDNF), neuronspecific enolase (NSE), S100- $\beta$ protein (S100- $\beta$ ), and nerve growth factor (NGF) were determined by ELISA. Oxidative stress indices including superoxide dismutase (SOD), malondialdehyde (MDA), and superoxide dismutase (GSH-PX) were determined by spectrophotometer method.

All the data were analysed by the Statistical Package for Social Sciences (SPSS) computer software programme (version 25). The levels of serum apoptotic factors, serum neurological function indices, serum oxidative stress indices were analysed by applying independent sample t-test for the detection of any significant differences between the treatment group and control group. The resulting data was expressed as mean \pm SD (standard deviation). A p-value of $<0.05$ was considered as significant.

\section{RESULTS}

Among the 90 newborns, 49 were males $(54.44 \%)$ and 41 were females (45.56\%); gestational age was $35-41$ weeks, with the mean gestational age of $38.15 \pm 2.76$ weeks; and birth weight was $2.41-3.92 \mathrm{Kg}$, with mean birth weight of $2.83 \pm 0.52 \mathrm{Kg}$. Disease grading SERNET Grading of HIE was mild in 52 cases $(57.78 \%)$, moderate in 34 cases $(37.78 \%)$, and severe in 4 cases $(4.44 \%)$. The mean gestational age of newborns in the treatment group was $38.06 \pm 2.95$ weeks, and 38.24 \pm 2.51 weeks in the control group.

After treatment, levels of serum PDCD5, sFas, and sFasL in the treatment group were lower than those in the control group (all $p<0.001$, Table I). Levels of serum BDNF and NGF in the treatment group were higher than those in the control group (both $p<0.001$ ). The levels of NSE and S100- $\beta$ in the treatment group were lower than those in the control group (both $p<0.001$, Table II). Levels of serum SOD and GSH-PX in the treatment group were higher than those in the control group (both $p<0.001)$. The content of MDA in the treatment group was lower than that in the control group $(p<0.001$, Table III).

Table I: Comparison of the levels of serum apoptotic factors after treatment between the two groups.

\begin{tabular}{l|c|c|c}
\hline Parameter & $\begin{array}{c}\text { Control group } \\
(\text { Mean } \pm \text { SD }) \\
\mathrm{n}=45\end{array}$ & $\begin{array}{c}\text { Treatment group } \\
(\text { Mean } \pm \text { SD }) \\
\mathrm{n}=45\end{array}$ & $\mathrm{p}$-value \\
\hline $\begin{array}{l}\mathrm{PDCD}(\mu \mathrm{g} / \mathrm{L}) \\
\mathrm{n}=45\end{array}$ & $10.64 \pm 1.66$ & $6.25 \pm 0.58$ & $<0.001$ \\
\hline $\mathrm{sFas}(\mu \mathrm{g} / \mathrm{L})$ & $18.57 \pm 2.61$ & $10.16 \pm 1.69$ & $<0.001$ \\
$\mathrm{n}=45$ & $4.14 \pm 1.05$ & $2.07 \pm 0.52$ & $<0.001$ \\
\hline $\mathrm{sFasL}(\mu \mathrm{g} / \mathrm{L})$ & & & \\
$\mathrm{n}=45$ & & & \\
\hline
\end{tabular}

SD=Standard deviation. 
Table II: Comparison of the levels of serum neurological function indices after treatment between the two groups.

\begin{tabular}{l|c|c|c}
\hline Parameter & $\begin{array}{c}\text { Control group } \\
(\text { Mean } \pm \text { SD }) \\
n=45\end{array}$ & $\begin{array}{c}\text { Treatment group } \\
(\text { Mean } \pm \text { SD }) \\
n=45\end{array}$ & p-value \\
\hline $\begin{array}{l}\text { BDNF }(\mu \mathrm{g} / \mathrm{L}) \\
\mathrm{n}=45\end{array}$ & $1396.21 \pm 169.22$ & $1823.85 \pm 241.56$ & $<0.001$ \\
\hline $\mathrm{NSE}(\mu \mathrm{g} / \mathrm{L})$ & $2.37 \pm 0.34$ & $1.18 \pm 0.57$ & $<0.001$ \\
$\mathrm{n}=45$ & $121.45 \pm 36.56$ & $146.39 \pm 24.11$ & $<0.001$ \\
$\mathrm{~S} 100-\beta(\mu \mathrm{g} / \mathrm{L})$ & & & $<0.001$ \\
$\mathrm{n}=45$ & & & \\
$\mathrm{NGF}(\mu \mathrm{g} / \mathrm{L})$ & & &
\end{tabular}

SD=Standard deviation.

Table III: Comparison of the levels of serum oxidative stress indices after treatment between the two groups.

\begin{tabular}{l|c|c|c}
\hline Parameter & $\begin{array}{c}\text { Control group } \\
\text { (Mean } \pm \text { SD) } \\
\mathrm{n}=45\end{array}$ & $\begin{array}{c}\text { Treatment group } \\
(\text { Mean } \pm \text { SD) } \\
\mathrm{n}=45\end{array}$ & $\mathrm{p}$-value \\
\hline $\begin{array}{l}\mathrm{SOD}(\mathrm{U} / \mathrm{L}) \\
\mathrm{n}=45\end{array}$ & $73.51 \pm 6.03$ & $86.85 \pm 6.52$ & $<0.001$ \\
\hline $\begin{array}{l}\mathrm{MDA}(\mathrm{mol} / \mathrm{L}) \\
\mathrm{n}=45\end{array}$ & $8.32 \pm 0.55$ & $5.74 \pm 0.49$ & $<0.001$ \\
$\begin{array}{l}\mathrm{GSH}-\mathrm{PX}(\mathrm{U} / \mathrm{mL}) \\
\mathrm{n}=45\end{array}$ & $38.53 \pm 4.51$ & $53.12 \pm 5.10$ & $<0.001$ \\
\hline SD=Standard deviation & & & \\
\hline
\end{tabular}

\section{DISCUSSION}

The purpose of clinical treatment of HIE is to maintain internal environment stability, improve damaged neurons, control convulsions, and promote cerebral circulation as much as possible. To find a reasonable and effective therapeutic drug for HIE is the focus of current research and treatment. Monosialoganglioside is neuroprotective agents that are widely found in the cell membrane nervous system of mammalian. Some studies have confirmed that monosialoganglioside has the ability to promote functional recovery of CNS injury caused by various causes, and possesses significant protection against neurodegeneration caused by injury. ${ }^{13,14}$ In addition, studies have also found that monosialoganglioside has the ability to protect against excitatory amino acid toxicity; and reduce cell death, and brain edema. ${ }^{15}$ The mechanism of protection and repair of damaged nerves by monosialoganglioside mainly manifests in many aspects. Monosialo-ganglioside can promote the growth of nerve cords, activate neurotrophic factors, reduce the death of cerebral neurons, cerebral cortical neurons, hippocampal pyramidal neurons and nerve cells, and inhibit damage to neurons by toxic products. ${ }^{16}$ Monosialoganglioside can protect calcium pumps, reduce intracellular calcium overload caused by calcium influx, protect cerebral ischemia, reduce edema, and promote recovery of damaged neuronal structures and functions. 17 In this study, monosialoganglioside was used as adjunctive therapeutics in children with HIE in our hospital. The role of monosialoganglioside was studied from the perspective of serological indicators.
Apoptosis and local neurological impairment caused by ischemia and hypoxia are the basis for the development of HIE. PDCD5, sFas, and sFasL are recognized as proapoptotic factors. ${ }^{18}$ In this study, the authors found that the levels of serum PDCD5, sFas, and sFasL in the treatment group were lower than those in the control group; which indicated that after treatment, the degree of neuronal apoptosis decreased in the treatment group, and monosialoganglioside had a better anti-apoptotic effect than citicoline.

Some studies have suggested that monosialo-ganglioside can reduce the release of excitatory amino acids such as amino acids which can have a direct killing effect on nerve cells; consequently, it is speculated that monosialoganglioside plays a role in anti-apoptosis of neural cells by this way. ${ }^{19}$ Apoptosis of nerve cells will directly cause neurological impairment in children with HIE. Both BDNF and NGF have the function of nourishing nerves and promoting the growth of axons. ${ }^{20}$ The levels of BDNF and NGF were significantly decreased in children with HIE, which was a direct sign of neurological impairment in children. NSE and S100- $\beta$ have very low serological levels in physiological conditions. They exist only in nerve cells and are released into the extracellular space when neuronal damage occurs. They enter the peripheral blood through the blood-brain barrier and the changes in the content are detected. In this study, it was found that the levels of serum BDNF and NGF in the treatment group were higher, and the levels of NSE and S100- $\beta$ were lower, which suggested that monosialoganglioside had neurotrophic effects of promoting nerve regeneration and reducing nerve cell destruction. This conclusion is also consistent with previous findings. ${ }^{21}$

Intracellular calcium overload in neurons and the resulting damage to oxygen-free radicals have been one of the most important causes of the exacerbation of HIE and the expansion of nerve injury. Effective clearance of oxygen-free radicals in children is one of the keys to HIE therapy. 22 In this study, the content of oxidative stress indices in the two groups was detected. It was found that the level of serum MDA was lower and the levels of SOD and GSH-PX were higher in the treatment group after treatment, which revealed that the oxidation / antioxidation balance system in children with HIE was optimized after treatment with monosialoganglioside.

The limitations of this study relate to the sample size and focus on only the biochemical effects. The neurological outcome of the newborns with HIE, and outcome of these neonates and correlation with the lab findings need further exploration and research.

\section{CONCLUSION}

Monosialoganglioside can effectively improve the apoptotic factors, neurological function, and oxidative 
stress indices in newborns and maintain the stability of the internal environment, so it is worthy of promotion and application.

\section{REFERENCES}

1. Weems MF, Upadhyay K, Sandhu HS. Survey of ECMO practices for infants with hypoxic ischemic encephalopathy. J Perinatol 2018; 38:1197-204.

2. Butt TK, Farooqui $R$, Khan MA. Risk factors for hypoxic ischemic encephalopathy in children. $J$ Coll Physicians Surg Pak 2008; 18:428-32.

3. Itoo BA, Al-Hawsawi ZM, Khan AH. Hypoxic ischemic encephalopathy. Incidence and risk factors in North Western Saudi Arabia. Saudi Med J 2003; 24:147-53.

4. Martinello K, Hart AR, Yap S, Mitra S, Robertson NJ. Management and investigation of neonatal encephalopathy: 2017 update. Arch Dis Child Fetal Neonatal Ed 2017; 102:F346-58

5. Chalak L, Latremouille S, Mir I, Sánchez PJ, Sant'Anna G. A review of the conundrum of mild hypoxic-ischemic encephalopathy: Current challenges and moving forward. Early Hum Dev 2018; 120:88-94.

6. Ahmad QM, Chishti AL, Waseem N. Role of melatonin in management of hypoxic ischaemic encephalopathy in newborns: A randomized control trial. J Pak Med Assoc 2018; 68:1233-7.

7. Ertl M, Weber S, Hammel G, Schroeder C, Krogias C. Transorbital sonography for early prognostication of hypoxicischemic encephalopathy after cardiac arrest. $J$ Neuroimaging 2018; 28:542-8.

8. Lu J, Yao XQ, Luo X, Wang Y, Chung SK, Tang HX, et al. Monosialoganglioside 1 may alleviate neurotoxicity induced by propofol combined with remifentanil in neural stem cells. Neural Regen Res 2017; 12:945-52.

9. Li S, Xiao N, Zhang X, Liu L, Lin L, Chen S, et al. Effects of exogenous ganglioside-1 on learning and memory in a neonatal rat model of hypoxia-ischemia brain injury. Neural Regen Res 2008; 3:1004-9.

10. Cohen RA, Browndyke JN, Moser DJ, Paul RH, Gordon N, Sweet L. Long-term citicoline (cytidine diphosphate choline) use in patients with vascular dementia: neuroimaging and neuropsychological outcomes. Cerebrovasc Dis 2003; 16: 199-204.
11. Conant R, Schauss AG. Therapeutic applications of citicoline for stroke and cognitive dysfunction in the elderly: A review of the literature. Altern Med Rev 2004; 9:17-31.

12. Giménez R, Soler S, Aguilar J. Cytidine diphosphate choline administration activates brain cytidine triphosphate: phosphocholine cytidylytransferase in aged rats. Neurosci Lett 1999; 273:163-6.

13. Samson JC. GM1 ganglioside treatment of central nervous system injury: Clinical evidence for improved recovery. Drug Develop Res 1990; 19:209-24.

14. Stephen D. Skaper, Alberta Leon, Gino Toffano. Ganglioside function in the development and repair of the nervous system. Mol Neurobiol 1989; 3:173-99.

15. Skaper SD, Leon A. Monosialogangliosides, neuroprotection, and neuronal repair processes. J Neurotrauma 1992; 9: S507-16.

16. Abou-Abbass H, Bahmad H, Abou-El-Hassan H, Zhu R, Zhou S, Dong $\mathrm{X}$, et al. Deciphering glycomics and neuroproteomic alterations in experimental traumatic brain injury: Comparative analysis of aspirin and clopidogrel treatment. Electrophoresis 2016; 37:1562-76.

17. Sheng L, Li Z. Adjuvant treatment with monosialoganglioside may improve neurological outcomes in neonatal hypoxicischemic encephalopathy: A meta-analysis of randomized controlled trials. PloS One 2017; 12:e0183490.

18. Tracy CM, Gray AJ, Cuéllar J, Shaw TS, Howlett AC, Taylor RM, et al. Programmed cell death protein 5 interacts with the cytosolic chaperonin containing tailless complex polypeptide 1 (CCT) to regulate $\beta$-tubulin folding. J Biol Chem 2014; 289:4490-502.

19. Facci L, Leon A, Skaper SD. Hypoglycemic neurotoxicity in vitro: involvement of excitatory amino acid receptors and attenuation by monosialoganglioside GM1. Neuroscience 1990; 37:709-16.

20. Oliveira NK, Ferreira RN, Lopes S, Chiari E, Camargos E, Martinelli PM. Cardiac autonomic denervation and expression of neurotrophins (NGFand BDNF) and their receptors during experimental chagas disease. Growth Factors 2017; 35:161-70.

21. Gorria M, Huc L, Sergent O, Rebillard A, Gaboriau F, Dimanche-Boitrel MT, et al. Protective effect of monosialoganglioside gm1 against chemically induced apoptosis through targeting of mitochondrial function and iron transport. Biochem Pharmacol 2006; 72:1343-53.

22. Singh SK, Dua T, Tandon A, Kumari S, Ray G, Batra S. Status of lipid peroxidation and antioxidant enzymes in hypoxic ischemic encephalopathy. Indian Pediatr 1999; 36:561-6. 